\title{
Ontogenetic and phylogenetic simplification during white stripe evolution in clownfishes
}

\author{
Pauline Salis ${ }^{1}$, Natacha Roux ${ }^{1}$, Olivier Soulat ${ }^{2}$, David Lecchini ${ }^{3}$, Vincent Laudet ${ }^{*}$ and Bruno Frédérich ${ }^{4}$
}

\begin{abstract}
Background: Biologists have long been fascinated by the striking diversity of complex color patterns in tropical reef fishes. However, the origins and evolution of this diversity are still poorly understood. Disentangling the evolution of simple color patterns offers the opportunity to dissect both ultimate and proximate causes underlying color diversity.

Results: Here, we study clownfishes, a tribe of 30 species within the Pomacentridae that displays a relatively simple color pattern made of zero to three vertical white stripes on a dark body background. Mapping the number of white stripes on the evolutionary tree of clownfishes reveals that their color pattern diversification results from successive caudal to rostral losses of stripes. Moreover, we demonstrate that stripes always appear with a rostral to caudal stereotyped sequence during larval to juvenile transition. Drug treatments (TAE 684) during this period leads to a dose-dependent loss of stripes, demonstrating that white stripes are made of iridophores and that these cells initiate the stripe formation. Surprisingly, juveniles of several species (e.g., Amphiprion frenatus) have supplementary stripes when compared to their respective adults. These stripes disappear caudo-rostrally during the juvenile phase leading to the definitive color pattern. Remarkably, the reduction of stripe number over ontogeny matches the sequences of stripe losses during evolution, showing that color pattern diversification among clownfish lineages results from changes in developmental processes. Finally, we reveal that the diversity of striped patterns plays a key role for species recognition.
\end{abstract}

Conclusions: Overall, our findings illustrate how developmental, ecological, and social processes have shaped the diversification of color patterns during the radiation of an emblematic coral reef fish lineage.

Keywords: Ontogeny, Diversification, Pomacentridae, Color evolution, Adaptive coloration

\section{Background}

Understanding the diversification of phenotypes requires to integrate developmental and evolutionary analysis in an ecological context [1]. Having a well-defined phylogenetic context is essential to recognize the pattern of trait evolution as well as to detect events of parallel or convergent evolution. In addition, studying how phenotypic traits differ across natural environments as well as their adaptive value allows to reveal the factors shaping the emergence of diversity. Lastly, the study of trait

\footnotetext{
* Correspondence: vincent.laudet@obs-banyuls.fr

1Observatoire Océanologique de Banyuls-sur-Mer, UMR CNRS 7232 BIOM,

Sorbonne Université Paris, 1, Avenue Pierre Fabre, 66650 Banyuls-sur-Mer,

France

Full list of author information is available at the end of the article
}

development helps to identify the molecular mechanisms behind phenotypic diversification as well as constraints that bias their evolutionary trajectories.

Pigmentation, in particular color patterns, provides an incredible number of cases that allow the exploration of the interplay between ecology, evolution, and development that are at the basis of trait diversification [2-6]. Among vertebrates, coral reef fishes provide classical examples of complex color patterns exhibiting a huge variety, and therefore, they offer a unique opportunity to better understand, in an integrated manner, the origin of those traits [7]. Most of coral reef fish species display spots, stripes, repeated lines, eyespots, grids, etc. This diversity in color patterns serves for species recognition $[8,9]$, camouflage $[10,11]$, mimicry [12], and/or warning [13]. For example, 
the eyespots of the damselfish Pomacentrus amboinensis have been suggested to serve as a subordinate signal directed to dominant males [14]. To date, work on coral reef fishes has mainly been focused on the link between color patterns, ecology, and behavior, that is, the ultimate role of these patterns [15]. However, the underlying development controlling these patterns and their evolution, that is, their proximal mechanism, is still largely unknown $[15,16]$.

It is now well known that phenotypic diversification between lineages may be achieved by changes in developmental processes $[1,17]$. There are a number of possible developmental mechanisms that explain how specific changes in signaling pathways can induce phenotypic changes between lineages, and a main goal of Evo/Devo is to better understand these processes. Within this framework, various studies devoted to the pigmentation of zebrafish allowed to pinpoint changes in developmental mechanisms leading to color variation among related fish species [18-20]. However, the incredibly diverse color patterns of coral reef fishes have never been explored with such an Evo/Devo perspective. Despite this, there are some evidences that developmental processes may indeed sustain the diversification of color patterns in some species. For example, the polymorphic damselfish Chrysiptera leucopoma may retain its juvenile color (a bright yellow body with a dorsal blue line) or shift to the adult phenotype (a dark brown body) depending on habitat type and/ or population densities [21]. However, in this example, no study of the underlying developmental mechanisms has been performed.

Clownfishes (Amphiprion and the monotypic Premnas) are iconic coral reef fishes [22]. This tribe (Amphiprionini; [23]) within Pomacentridae is composed of 30 species that display a relatively simple color pattern made of zero to three white vertical stripes that are well visible on a yellow to red, brown, or even black body background [22]. Their life cycle includes a relatively short dispersive planktonic larval phase in the open ocean [24], followed by the settlement of juveniles into sea anemones where they live in a social group composed of a dominant breeding pair and a varying number of sexually immature subalterns [22]. The functional role of striped patterns in clownfishes is still unknown but could be associated with predator defense, foraging mode, macro-habitat type, species recognition, etc. as observed in various teleosts $[15,25]$. The relatively simple color pattern of Amphiprion offers a good opportunity to better delineate the patterns and processes allowing the diversification of such ornamental diversity. The clownfish evolutionary radiation has recently received much attention, providing a suitable phylogenetic framework for testing new evolutionary hypotheses on the rise of color diversity in coral reef fishes [26].

In this study, we focus on the vertical white stripes present in most species of Amphiprion. We first map their occurrence and pattern on the clownfish evolutionary tree and reconstruct the ancestral state in terms of white stripe presence/absence. Our results provide evidences that the diversification of clownfish color pattern results from successive caudal to rostral losses of stripes during evolution. Using specific drugs (e.g., TAE 684: an inhibitor of tyrosine kinase receptors expressed in zebrafish iridophores), we reveal that the white stripes are formed by iridophores and are essential for the patterning of the neighboring black stripes. Then, by an ontogenetic approach, we show that either the juvenile has the same number of stripes than adults or the juvenile has supplementary stripes that disappear caudo-rostrally later on. The reduction of stripe number over ontogeny totally matches the sequences of stripe losses across evolution, demonstrating that the diversification in color pattern among clownfish lineages results from changes in developmental processes. Finally, we determine the links between the number of stripes and other external morphological traits, and we provide some evidence that the various striped patterns have evolved for species recognition. This approach allows us to consider the relationships among striped patterns, fish morphology, and their ecology to suggest that both developmental and ecological processes have shaped the diversity of color patterns in clownfishes.

\section{Results}

Successive caudo-rostral loss of stripes during evolution

Clownfishes can be classified into four categories according to their striped pattern at the adult stage: species without vertical stripe (group A) or species having one white vertical stripe (on the head-group B), two vertical stripes (on the head and the trunk-group C), or three vertical stripes (head, trunk, and caudal peduncle-group D) (Fig. 1 and Additional file 1: Table S1). Interestingly, there is no species with a single stripe on the trunk or on the peduncle (Fig. 1). White stripe on the trunk is always associated with a head stripe. The white stripe on the peduncle is always preceded by stripes on the head and the trunk.

To understand the evolution of color pattern in clownfishes, we performed a stochastic mapping of striped patterns on the most complete time-calibrated phylogeny of Amphiprionini [27]. The analysis highly suggests that the common ancestor of extended clownfishes exhibited three vertical white stripes $(90-100 \%$ of posterior probabilities; Fig. 2), independent from the color pattern polymorphism of some species (Additional file 2: Figure S1). The state reconstruction for every internal node of the phylogeny illustrates successive losses of vertical stripes from the caudal to the rostral region (Fig. 2). The stochastic mapping, which was performed with a model assuming that all transition rates are free to vary, 

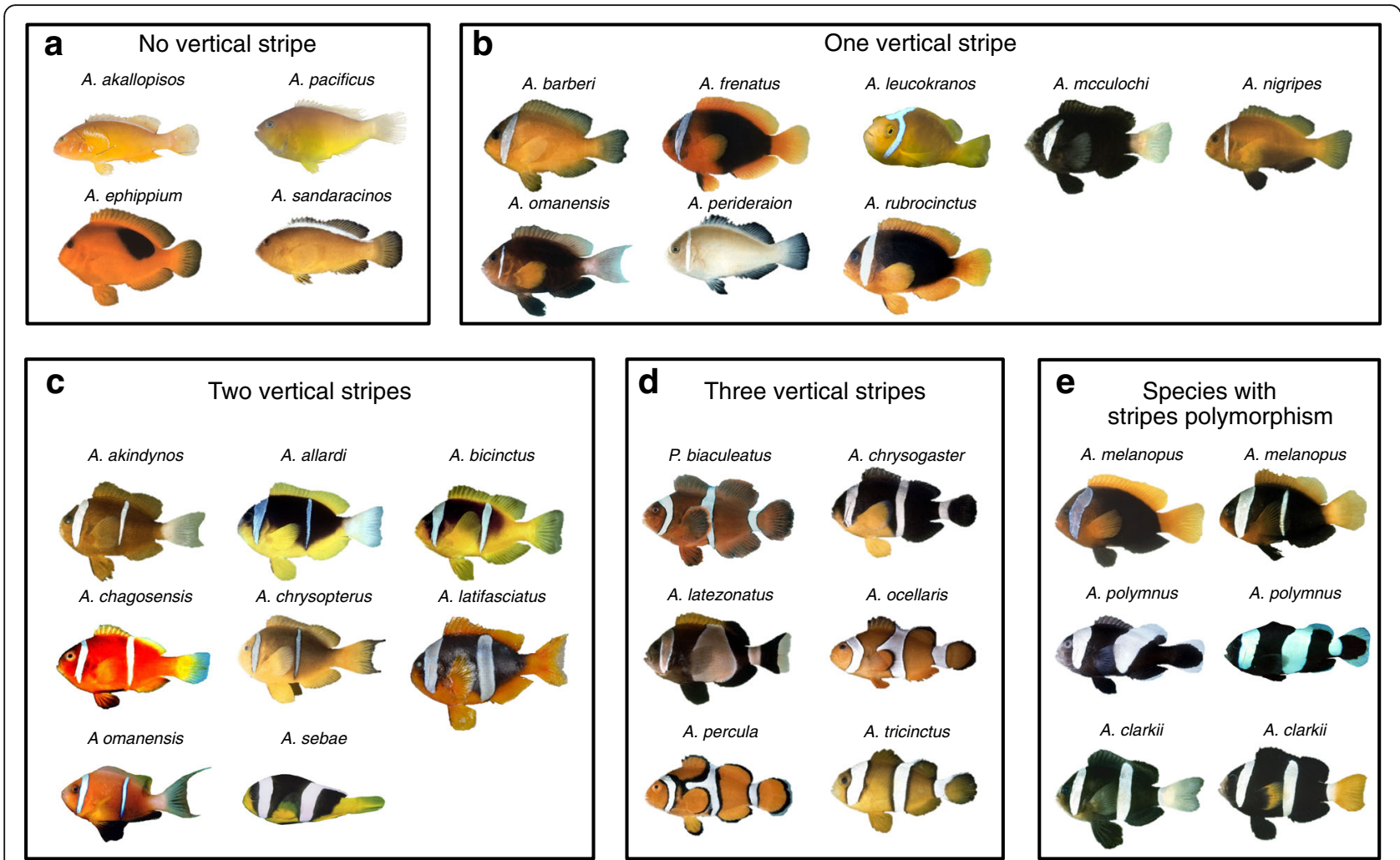

Fig. 1 Adult color patterns of clownfish species. Pictures of adult clownfishes classified depending on their color patterns. a No vertical stripe, b one vertical stripe on the head, $\mathbf{c}$ two vertical stripes (one on the head, the other on the body), $\mathbf{d}$ three vertical stripes (one on the head, one on the body trunk, and the last one on the peduncle), e fishes having stripes polymorphism

shows that some transitions between stripe morphs never occur. The evolutionary loss or gain of two white stripes appears very unlikely but the gain of one stripe, i.e., reversion, is possible (e.g., Amphiprion chrysogaster-Fig. 2 and Additional file 3: Table S2). Thus, we tested these hypotheses by comparing the fit of four evolutionary models varying in their matrix of transition rates between stripe patterns using the Multiple State Speciation Extinction (MuSSE) method [28, 29]. In these four models, rates of speciation and extinction were constrained to be equal among stripe morphs in order to reduce the number of model parameters. The best-fitting models according to the Akaike Information Criterion are the most constrained models iii and iv (Table 1 and Additional file 4: Table S3). Both models assume that the loss or the acquisition of two stripes is not allowed. Moreover, in the great majority of the scenarios tested (Additional file 4: Table S3), model fitting highly suggests that the transition rates among stripe morphs are symmetric, i.e., the rate shift from stripe morph $A$ to $B$ is equal to the rate shift from stripe morph $B$ to $A$. The estimation of transition rates from the best-supported model (model iv) suggests that the appearance/disappearance of the third stripe on the caudal peduncle occurred more slowly (mean $\pm \mathrm{SD}=0.052 \pm 0.001$ ) than the one of the second stripe in the trunk $(0.103 \pm 0.001)$ or the first in the head $(0.123 \pm 0.001)$.

This evolutionary analysis therefore highlights that the diversification of white stripe pattern in clownfishes is a history of loss from an ancestral lineage having three stripes and that these losses always occurred in a progressive and sequential fashion from caudal to rostral regions. For example, in all two striped species, the peduncle stripe has been lost and the head and the trunk stripes are retained. All one-stripe species retained the head stripe and have lost the peduncle and trunk stripes.

\section{Ontogeny of stripe formation reveals a rostro-caudal stereotyped pattern}

The fact that stripes always appear at the same location in clownfishes and that the losses of stripes during evolution occurred in a sequential manner from caudal to rostral suggests that this loss may be constrained by a developmental mechanism. We thus tested whether a variation in the number of stripes could occur during clownfish ontogeny by studying the development of Amphiprion ocellaris and Amphiprion frenatus that display three stripes (i.e., similar to the ancestral state) or a single head stripe at the adult stage, respectively. 


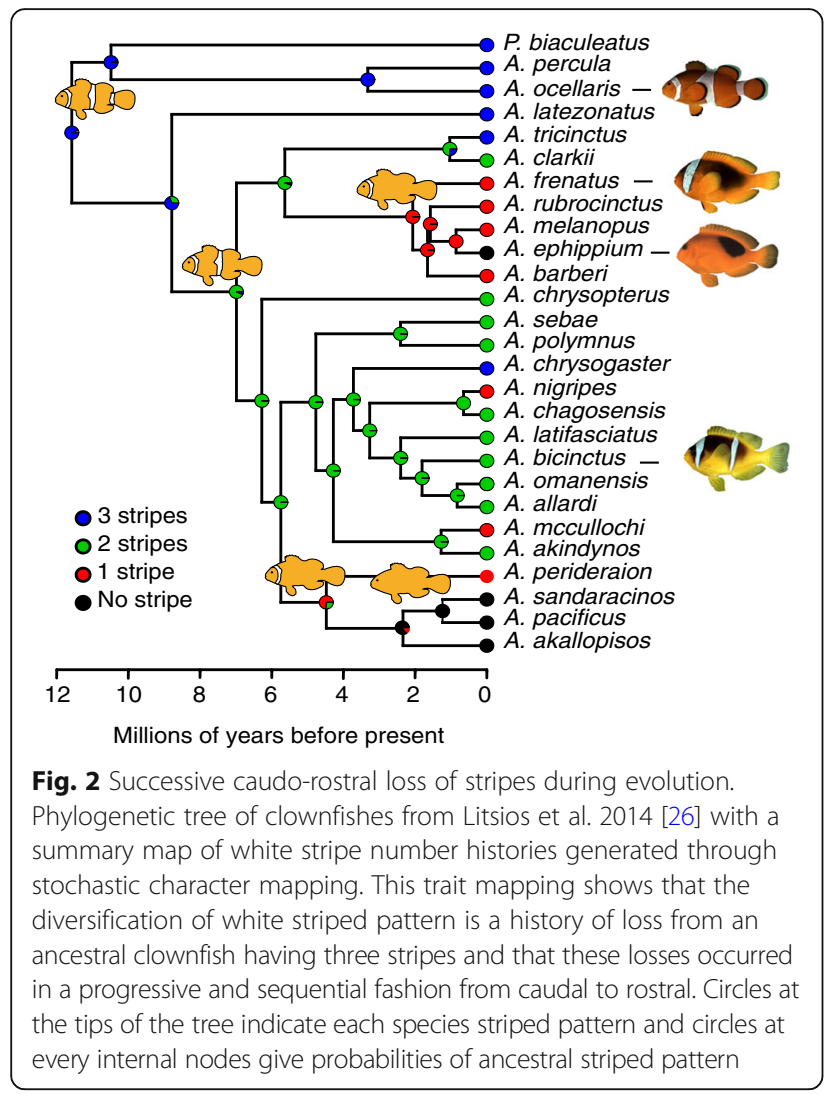

At 8 days post hatching (dph), larvae of both species do not harbor any vertical stripe (Fig. 3a-a' and d-d'). At $10-11 \mathrm{dph}, A$. ocellaris larvae acquire the head and trunk stripes simultaneously (Fig. 3b). Surprisingly, the same is true for A. frenatus (Fig. 3e-e'). In A. ocellaris, the third stripe on the caudal peduncle is formed at 14 dph (Fig. 3c). Strikingly, we also observed the development of a third stripe on the caudal peduncle of some $A$. frenatus at the same larval period, as in A. ocellaris (Fig. 3f). In our husbandry conditions, larvae of $A$. frenatus reach the juvenile stage with either two or three stripes (Fig. 3h): the anterior one on the head, the medial

Table 1 Model fitting of the four striped pattern evolutionary models

\begin{tabular}{lllll}
\hline & Transition matrix & AIC & $\triangle$ AIC & wtAIC \\
\hline Model iv & Model ii + model iii & 191.74 & 0.00 & 0.88 \\
Model iii & $\begin{array}{l}\text { Loss or gain of two stripes } \\
\text { are forced to be 0 }\end{array}$ & 196.58 & 4.84 & 0.08 \\
Model ii & $\begin{array}{l}\text { Symmetric rates of transition } \\
\text { among states }\end{array}$ & 197.74 & 6.00 & 0.04 \\
Model i & Free & 205.58 & 16.84 & 0.00
\end{tabular}

Models are ranked from best to worst, according to AIC scores and Akaike weights (wtAIC). $\triangle \mathrm{AIC}$ scores indicate the difference between the candidate model and the best-fitting model. According to [58], a $\triangle \mathrm{AICC}$ value of 4 or more was taken as an indication of support for one model over the other following. Matrices of transition rates (q) among stripe morphs are described for the four evolutionary models. Speciation and extinction rates are assumed to be equal among morphs one on the trunk, and the posterior one on the caudal peduncle whereas $A$. ocellaris reach the juvenile stage with three stripes (Fig. 3g). The loss of the trunk stripe will occur after several months and is therefore a prominent feature of juvenile $A$. frenatus.

Our data provide strong evidence that there are two distinct and inverse phenomena: (i) an evolutionary pattern of stripe loss, with a caudo-rostral progression that is observed in adult fish, and (ii) a developmental pattern of rostro to caudal stripe gain during the larval to juvenile transition exemplified in A. ocellaris and A. frenatus followed by a sequential loss of caudo-rostral during juvenile stage in some species such as $A$. frenatus. Taken together, these results emphasize the fact that clownfish color pattern evolution is constrained by developmental processes that may also explain why there is no species with a single stripe on the tail or trunk.

In order to better understand the white stripe ontogenesis, we examined the cellular process by which the stripes are formed. In teleost fishes, different types of pigment cells (or chromatophores) are described according to their ultrastructure and their pigment type [30]. Xanthophores (orange cells), iridophores (white and iridescent cells), leucophores (white), and melanophores (black cells) are the four main characterized cells. In $A$. ocellaris, we observed at least three types of cells: xanthophores, melanophores, and white cells (Fig. 4f). From juvenile to adult stage, orange skin is composed of xanthophores and round melanophores. The white stripe comprises stellar melanophores and white cells whereas the black stripes are formed of a dense number of melanophores (Fig. 4a). To determine if leucophores or iridophores are involved in white stripe formation in $A$. ocellaris, we tested whether the drug TAE684 (TAE), known as an inhibitor of leukocyte tyrosine kinase (Ltk) and anaplastic lymphoma kinase (Alk) and leading to a decrease of iridophore number in zebrafish [31], could disrupt white stripe formation. Treatments with TAE at $0.6 \mu \mathrm{M}$ or $0.3 \mu \mathrm{M}$ during the metamorphosis of $A$. ocellaris larvae (i.e., from 5 until $18 \mathrm{dph}$ ) induced a dose-response effect into the formation of white stripes (Fig. 4a-d). While the control develops the head, the trunk, and the peduncle white stripes (Fig. 4a), fish treated with $0.6 \mu \mathrm{M}$ TAE develop a complete transparent head stripe whereas the body stripe is uncompleted. This suggests that there is a decrease in the number of iridophores (Fig. 4d, e). Fish treated with $0.3 \mu \mathrm{M}$ show an intermediate phenotype with $50 \%$ of the fish having the same phenotype as fish treated with $0.6 \mu \mathrm{M}$ (Fig. 4c, e) and 50\% having full transparent head and trunk vertical stripes (Fig. 4b, e). Similarly, a dose-response effect on the iridescence of the eye was observed (Fig. 4 right panel a-d). Indeed, whereas the eyes of fish control and fish treated at 


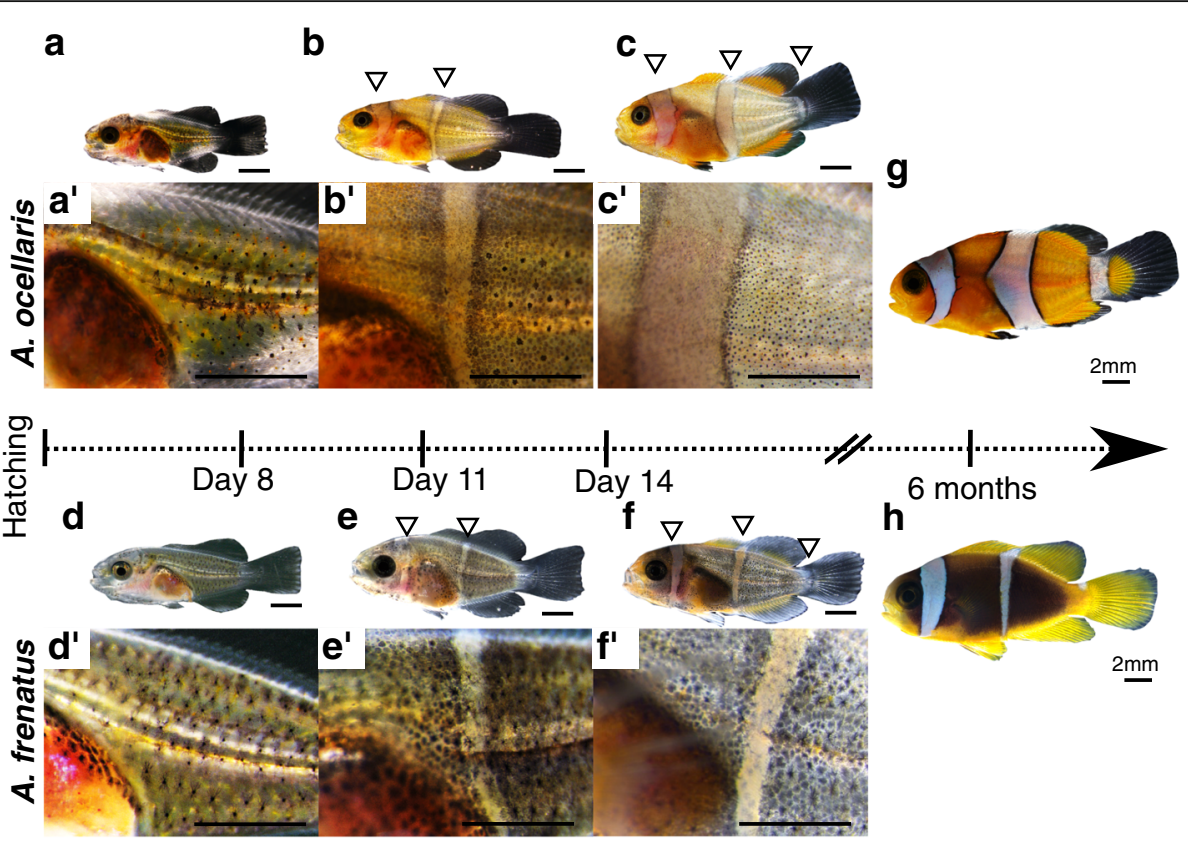

Fig. 3 Ontogeny of stripe formation reveals a rostro-caudal stereotyped pattern. A. ocellaris (a- $c^{\prime}$ and g) and A. frenatus (d-f' and $\left.h\right)$ color pattern ontogenesis at 8 dph (a-a', d-d', A. ocellaris: $n=10 ;$ A. frenatus: $n=3), 11$ dph (b-b', e-e', A. ocellaris: $n=10 ; A$. frenatus: $n=3), 14$ dph (c-c', f-f', A. ocellaris: $n=10 ;$ A. frenatus: $n=3$ ) and 6 months post hatching ( $g$ and $h, n=5$ ). Higher magnification of the medial white stripe ontogenesis $\left(a^{\prime}, b^{\prime}, c^{\prime}, d^{\prime}, e^{\prime}, f^{\prime}\right)$. Note that the white stripes appear in the same rostral to caudal sequence in both species. Scale bars correspond to $1 \mathrm{~mm}$

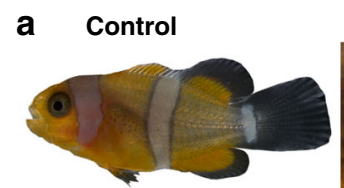

b TAE684 $0.3 \mu \mathrm{M}$

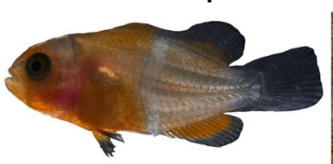

C TAE684 $0.3 \mu \mathrm{M}$

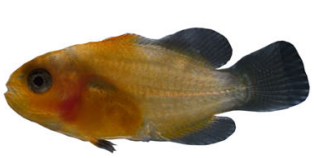

d TAE684 $0.6 \mu \mathrm{M}$

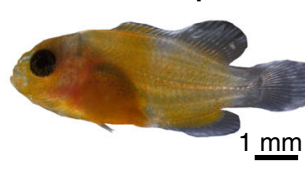

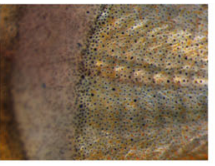
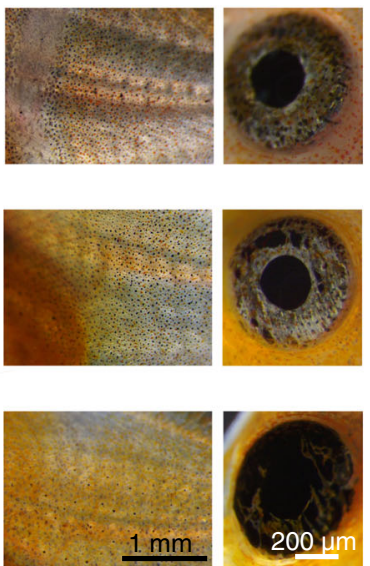
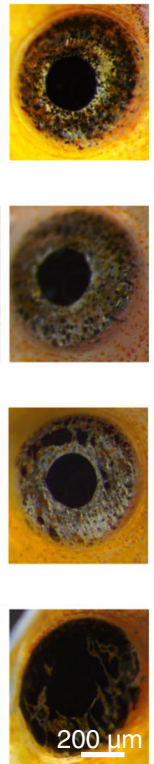

e

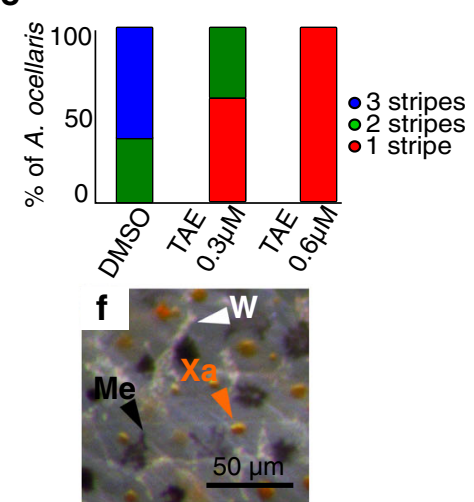

g $10 \mathrm{dph}$ h $11 \mathrm{dph}$

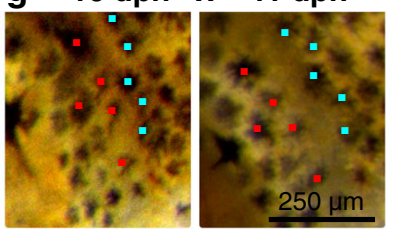

Fig. 4 Cellular mechanism of color pattern ontogenesis in A. ocellaris. a-d Dose-dependent modifications of color pattern (left and middle panel) and iridescence of the eye (right panel) after 13 days of TAE684 drug treatment of A. ocellaris at $18 \mathrm{dph}$ at $0.6 \mu \mathrm{M}(\mathbf{d})$ and $0.3 \mu \mathrm{M}(\mathbf{b}, \mathbf{c})$ compared to DMSO (control, a). e Cumulative histogram of fishes having fully stripes: one stripe (head-red), two stripes (head and trunk—green), or three stripes (blue) in control $(n=6)$, TAE $0.3 \mu \mathrm{M}(n=16)$ and TAE $0.6 \mu \mathrm{M}(n=3)$. f Stereomicroscope pictures showing the three types of chromatophores within the trunk of juvenile A. ocellaris. $(\mathbf{g}, \mathbf{h}, n=4)$. Live imaging pictures of the same A. ocellaris individual at $10 \mathrm{dph}(\mathbf{g})$ and $11 \mathrm{dph}(\mathbf{h})$ show that during medial white stripe formation, the distance increases between melanophores underlined with red dots and melanophores underlined with blue dots 
$0.3 \mu \mathrm{M}$ TAE are iridescent (Fig. 4a-c), those of fish treated at $0.6 \mu \mathrm{M}$ TAE are blackish (Fig. $4 \mathrm{~d}$ ). Together, these results demonstrate that white cells correspond to iridophores but also that Ltk and/or Alk is required for the formation of iridophores and color pattern during metamorphosis of A. ocellaris.

TAE treatments during the metamorphosis of A. ocellaris larvae allow the understanding of how iridophores contribute to color pattern development. White vertical stripes are surrounded by a thin stripe of melanophores over an orange body. Interestingly, we observe that when no white vertical stripe is formed over the body (Fig. 4d), melanophores are dispersed with xanthophores over the flank and they do not form any stripe. This is interesting to link to this observation that, during formation of the white stripe in control fish, iridophores initially appear at the future stripe location and push black melanophores at the periphery to form stripe pattern (Fig. $4 \mathrm{~g}, \mathrm{~h}$ ). This suggests that melanophores are expelled from the white stripe in normal condition to form the black stripes. In addition, we observed that xanthophores are not localized at their proper location after exposure with TAE. These data not only reveal that the cellular substrate underlying the white color is likely based on iridophore cells but also that they shed light on the fact that specific interactions between different chromatophore types play an important role in the stripe formation in A. ocellaris.

\section{Stripe loss during ontogeny occurs multiple times in Amphiprion}

The observation that $A$. frenatus juveniles have more stripes than adults prompted us to further document the evolution of ontogenetic trajectories of color pattern in clownfishes. For this, we compiled ontogenetic information on 26 Amphiprion species with a multi-tiered approach utilizing the primary literature, online databases, and field observations made by various experts (Additional file 1: Table S1). We observed that a minimum of nine species show extra stripes during the juvenile phase when compared to adult. This is illustrated on Fig. 5 for four species: A. frenatus, A. melanopus, A. rubrocinctus, and $A$. ephippium. This contrasts with other cases (e.g., $A$. nigripes or A. sandaracinos; Fig. 5e, f) for which the number of stripes is invariant over ontogeny. We therefore categorized the species showing a loss of stripes during ontogeny (group 1) and the species without a loss of stripes during their development (group 2) (Additional file 1: Table S1), and we studied the evolution of these two trajectories during the evolution of clownfishes (Fig. 5g). Ancestral state reconstruction supports (64\% of posterior probabilities) that the last common ancestor of extended clownfishes did not lose white stripes during ontogeny. Moreover, stochastic mapping reveals a minimum of five major transitions in the occurrence of white stripe loss during ontogeny: (i)
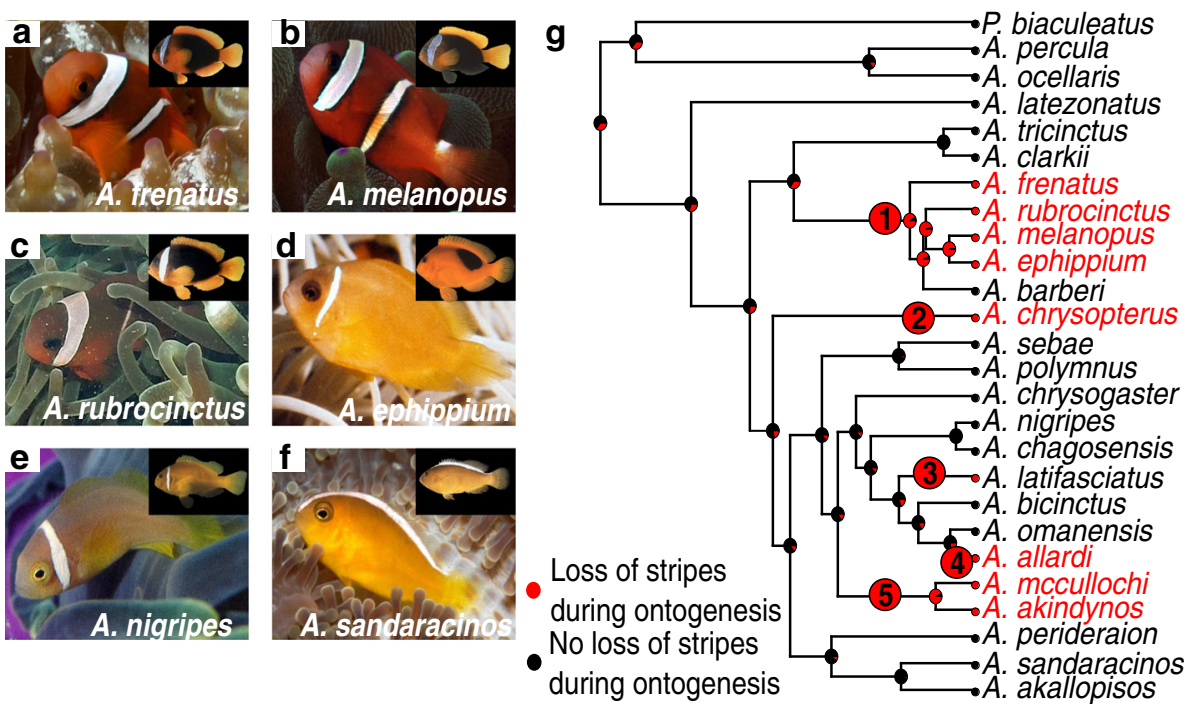

during ontogenesis

Fig. 5 Stripe loss during ontogeny occurs multiple times in Amphiprion. a-f Pictures of juveniles (big picture) and adult (small picture-top right) of A. frenatus (a), A. melanopus (b), A. rubrocinctus (c), A. ephippium (d), A. nigripes (e), and A. sandaracinos (f). A. frenatus (a), A. melanopus (b), A. rubrocinctus (c), and A. ephippium (d) show that juveniles have extra stripes compared to its respective adult whereas the number of vertical stripes does not vary over ontogeny in A. nigripes (e) and A. sandaracinos (f). Pictures of juveniles were nicely provided by GR Allen. $\mathbf{g}$ Maximum clade credibility phylogeny of clownfishes [27] with a summary map of striped pattern ontogenesis generated through stochastic character mapping. It reveals a minimum of five major transitions to an ontogenetic pattern made of white stripe loss, occurring (1) in the $A$. frenatus clade, (5) in the clade grouping A. mccullochi and A. akindynos, and in three individual species (2) A. chrysopterus, (3) A. latifasciatus, and (4) A. allardi (number in red circles) 
one in the frenatus clade, (ii) one in A. chrysopterus, (iii) one in A. latifasciatus, (iv) one in A. allardi, and (v) one in the clade grouping $A$. mccullochi and $A$. akindynos (Fig. 5g, red circles). This reveals a convergence in the process of loss of stripes during ontogeny, probably triggered by selective factors (ecological, behavioral, etc.), and/or shared molecular and cellular mechanisms.

\section{Links between striped patterns, ecology, and external morphology}

Striped patterns are adaptive and related to ecological and behavioral differences among cichlid species [25]. In butterflyfishes, striped body patterns showed correlated evolution with a number of ecological factors including habitat and sociality [15]. Clownfishes vary in their ecology [22], and one of the most striking variations among species is the diversity of sea anemone hosts. Some clownfishes are specialists, living with only one sea anemone species, while some others are generalists, capable of living in association with several host species [32]. To establish if striped patterns of clownfishes are related to this ecological difference, we first tested the simple prediction that the number of white stripes is related to the number of possible sea anemone hosts using phylogenetic generalized least-squares (PGLS) regressions. However, the number of white stripes is unrelated to the number of hosts in which they exhibit mutualistic interactions $(F=0.13, P=0.72$; Additional file 5: Table S4).

Clownfishes also vary in their external morphology (Fig. 6), and it was suggested that this morphological disparity is related to variation in both host type and habitat partitioning [33]. At a first glance, the shape of the dorsal fin varies among clownfish species according to their white stripe patterns (Fig. 6). Indeed, an indentation at the middle of the dorsal fin in clownfish species having two and three stripes is visible (Fig. 6a, b, e-g) whereas this is less obvious in species having one and zero stripes (Fig. 6c, e-g and Additional file 6: Figure S2). Thus, we focus on fish body form, knowing that the number of color stripes might be size-dependent [34] and fin morphologies since these traits are usually linked to adaptation towards different macro-habitats (e.g., [35, 36]). We quantified body size, body elongation, and dorsal fin morphology of a minimum of 22 clownfish species and we tested whether stripe patterns are correlated with these morphological traits. Phylogenetic generalized

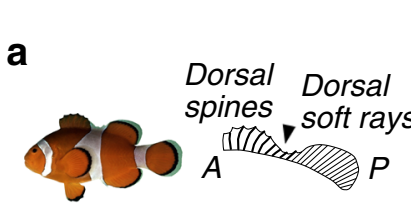

A. ocellaris

b
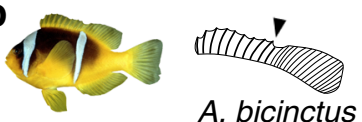

C
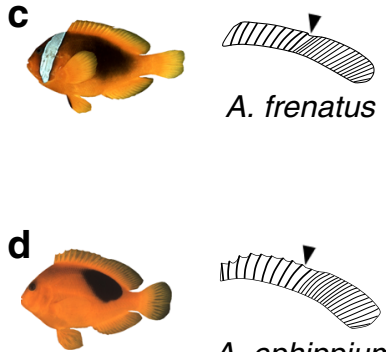

A. ephippium e Anterior lobe morphology index $($ I1-I2)/L

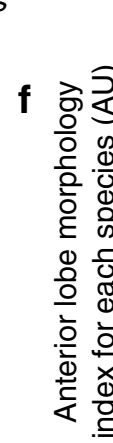

11
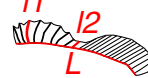

Posterior lobe
morphology index

$(\mathrm{Ir}-\mathrm{I} 2) / \mathrm{L}$

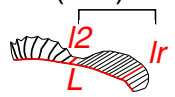

9

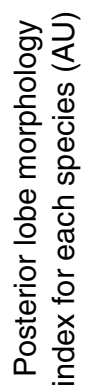

Fig. 6 Morphological trait analysis reveals a link between striped pattern and shape of the dorsal fin. a-d Pictures of A. ocellaris (a), A. bicinctus (b), A. frenatus (c), and A. ephippium (d) and cartoons illustrating their dorsal fin shape (A anterior, P posterior). There is an indentation at the middle of the dorsal fin (black arrowhead) with the anterior spiny rays longer than the most posterior one in clownfishes having two or three stripes. e Methods for quantification of anterior lobe and posterior lobe morphology index (11 length of the third dorsal spine, 12 length of the most posterior spine, Ir length of the longest soft ray, L length of the dorsal fin was used for normalization). Anterior and posterior lobes morphology indexes correspond to $(11-12) / L$ and $(I r-12) / L$, respectively. $\mathbf{f}, \mathbf{g}$ Scatterplots showing the relationship between the numbers of vertical white stripes ( $x$-axis) and lobe morphologies index of the dorsal fin (y-axis). Each point corresponds to one clownfish species 
least squares (PGLS) reveal that the evolution of the number of white stripes is unrelated to body size $(F=0.03$, $P=0.87)$ and body elongation $(F=0.64, P=0.43$; Additional file 5: Table S4). On the other hand, PGLS analysis shows a strong correlation between the anterior lobe morphology of the dorsal fin and the number of white stripes $(F=14.53, P<0.001)$ whereas the co-evolution of posterior lobe morphology and the number of white stripes is weaker $(P=0.05$; Additional file 5 : Table S4).

These analyses highlight that if there are few links between the striped pattern and clownfish body morphology, there is one between the striped pattern and dorsal fin shape. This link suggests that both traits depend on linked developmental and/or selective processes.

\section{Are striped patterns used for species recognition?}

"Species recognition" refers to the behavior whereby individuals identify and keep track of conspecifics for group coherence or identify a suitable sexual partner [37]. Accordingly, we hypothesized that the striped patterns of clownfishes could function for "species recognition," discouraging association of non-conspecifics and/ or encouraging association of conspecifics. Application of this hypothesis predicts that sympatric species should have distinctly different striped patterns. To test this hypothesis, we counted the number of identical pairs within eight communities of sympatric clownfishes [38] and we investigated whether similarity of striped patterns among sympatric species was less than expected if communities were composed of a random set of species. Relative to the four striped patterns, the diversity of clownfish communities is consistent with this species recognition hypothesis (Table 2). Indeed, except for The Keppels (Great Barrier Reef, Australia) and Komodo, the number of identical pairs was minimal within each natural community (Table 2). Moreover, the randomization test showed that this result is unexpected in the great majority of locations given the distribution of striped patterns among species. This test only failed for Keppels and Komodo communities, which house the highest number of polymorphic species (A. clarkii and A. melanopus). Overall, these data provide evidence that the distribution of striped patterns is not random in clownfish communities, suggesting that stripes help for species recognition.

\section{Discussion}

Our study reveals an unexpected link between the variation in the number of stripes occurring during ontogeny and the same type of variation occurring during evolution of clownfishes. Strikingly, the sequence of events observed during evolution mirror the one seen during ontogeny. An identical sequence of white stripe loss than the one observed during evolution was observed during late juvenile stages. These observations strongly suggest that the diversification of stripe patterns observed during clownfish radiation is the product of modifications of an ancestral stereotyped ontogenetic trajectory. From a mechanistic point of view, such a successive gain of stripes from the head to the caudal region during ontogeny support the hypothesis that loss of stripes during evolution is likely constrained by ontogenesis.

The sequential appearance of white stripes from the anterior to the posterior region during the development of two distantly related species, $A$. ocellaris and A. frenatus, is remarkable. This highly suggests a conserved mechanism of color pattern ontogeny across clownfishes. Here, we provide a first analysis into the mechanistic underpinnings of the patterns seen. In a first part, using an inhibitor of two receptor tyrosine kinases (Ltk and Alk) that are instrumental in iridophore formations in zebrafish, we show that the white coloration of stripes is produced by iridophores and not leucophores. After TAE treatments, we show that stripes are sometimes absent, incomplete, and/or less white. Disrupting the white stripe formation reveals that the presence of iridophores is instrumental for

Table 2 Diversity of clownfish communities is consistent with the species recognition hypothesis

\begin{tabular}{|c|c|c|c|c|c|c|}
\hline \multirow[t]{2}{*}{ Location } & \multirow[t]{2}{*}{ Number of species } & \multirow[t]{2}{*}{ Maximum of identical pairs } & \multicolumn{3}{|l|}{ Randomization } & \multirow[t]{2}{*}{$P$ value } \\
\hline & & & Proportion less & Proportion equal & Proportion greater & \\
\hline Marshall Island & 4 & 1 & 0.1393 & 0.6402 & 0.2205 & $<0.0001$ \\
\hline Japan & 3 & 0 & 0.1845 & 0.5715 & 0.2440 & $<0.0001$ \\
\hline Lombok & 3 & 0 & 0.1850 & 0.5676 & 0.2474 & $<0.0001$ \\
\hline Christmas Island & 3 & 0 & 0 & 0.4472 & 0.5528 & $<0.0001$ \\
\hline Bali & 3 & 0 & 0 & 0.4568 & 0.5432 & $<0.0001$ \\
\hline Komodo & 5 & 3 & 0.5958 & 0.3068 & 0.0974 & 1.00 \\
\hline Keppels (GBR) & 3 & 3 & 0.5131 & 0.2974 & 0.1895 & 1.00 \\
\hline Hoga & 6 & 3 & 0.1807 & 0.4369 & 0.3824 & $<0.0001$ \\
\hline
\end{tabular}

Number of pairs of identical stripe patterns in the eight clownfish communities, compared to communities assembled randomly from all clownfish species living in the western and central Pacific Provinces (i.e., a total of 10 species). The maximum number of identical pairs within each natural community was calculated by including the possibility of color polymorphism in some species 
the distribution of the two other chromatophore types. In TAE-treated fish, xanthophores and melanophores are scattered on the flank and not properly organized as in wild type. This suggests that, as in zebrafish [39], cell-cell interactions are critically important for pattern generation in clownfish. Additionally, our results suggest that clownfish color pattern is not formed by a reaction/diffusion mechanism based on Turing model [40] as in zebrafish. Up to now, our knowledge of stripe formation in teleost fishes derived from studies achieved in zebrafish, the most widely used fish model species (reviewed in [41]). However, it is likely that the pattern of stripe formation in zebrafish and clownfishes are controlled by different mechanisms. In zebrafish, the color adult pattern composed of periodical horizontal blue and orange stripes is effectively formed by a reaction/diffusion mechanism which predicts the periodic pattern [40]. This model is able to regulate the width of the stripes and it explains that, during growth, new stripes insert between the preexisting ones to keep stripe width [42]. Such Turing-like model was confirmed in long-fin zebrafish mutant which continues to form perfectly new stripes as the fins grow [43] and during normal growth of Pomacanthus imperator [44]. The case of clownfishes is totally different since the number of stripes is fixed and independent of fish body size. Moreover, in clownfishes, new stripes do not form when the distance between two previous ones increases but following an ordered anterior-to-posterior pattern. In addition, the disappearance of stripes during growth that we observed in some clownfish species (e.g., A. frenatus and $A$. chrysopterus) does not fit with Turing predictions and thus allows a clear refutation of the "Turing pattern hypothesis." This suggests that, in clownfishes, when and where the stripes must be formed are controlled by specific patterning mechanisms that remain to be analyzed.

Various clownfish species exhibit extra stripes during the juvenile phase when compared to the adult stage, and this convergent loss of stripes occurred at least five times across the evolution of clownfishes (Fig. 5). Such a convergence may be explained by shared molecular and cellular mechanisms triggering signals of stripe loss during ontogeny and/or by shared responses to selective factors (ecological, behavioral, etc.). In terms of proximal mechanisms, this loss of white stripes likely does not involve a modification of an ancestral pre-pattern but could result from different mechanisms. In zebrafish, it is known that pigment cells must be continuously formed to maintain the pattern of bands $[45,46]$. The loss of white stripe observed in some clownfish species during late juvenile life may be caused by a spreading of white iridophores over the body, an extensive apoptosis of these cells, and/or a dedifferentiation of the chromatophores resulting in an absence of pigment cell synthesis.
The correlated evolution between the numbers of vertical white stripes and dorsal fin shape suggests that both phenotypic traits may depend on the same or linked developmental processes. An ecomorphological interpretation of these results would be that patterns of stripes are somehow linked to differences in macro-habitats, but this hypothesis certainly needs further analyses. On the other hand, the fact that the white stripe is present mostly in species with an indentation in the dorsal fin may suggest that it may be linked to a disruptive effect [47], the band and indentation both helping to hide the fish silhouette. This may be part of a general strategy of poor swimmer fish to reinforce their disruptive coloration to avoid being tackled by predators. However, these observations urge for additional studies in order to disentangle the relationship between the color patterns and the ecology of clownfish species.

Beyond ecological adaptation, we provide evidence that the striped patterns play a role for species recognition in clownfishes. Indeed, the number of species pairs showing the same striped pattern is exceptionally low in natural communities compared with random ones. Non-significant results from Keppels and Komodo (Table 2) are probably due to stripe polymorphism of species living in these locations, a character which was included by default in our analyses. We hypothesize that this polymorphism of stripe numbers is likely driven by the function of species recognition but this needs to be tested. The diversity of white stripe patterns may result from social selection, where signals encourage association of conspecifics [48]. Indeed, social selection on visual signals can be very strong in clownfishes, which live in social groups based on a size dominance hierarchy where agonistic interactions are numerous [49-51]. We expect that a variation in the number of stripes during ontogenesis among individuals of a same species forming a social group may mediate or reduce agonistic interactions, ultimately facilitating the access for breeding positions [49]. The cohabitation of different clownfish species in the same sea anemone host may also support this hypothesis. Indeed, cohabiting clownfishes differ morphologically and always show dissimilar white stripe patterns [38]. While this hypothesis needs to be further explored, interspecific signaling and social selection on visual signals probably operate on the phenotypic divergence of clownfishes.

\section{Conclusion}

Our study highlights a strong link between the diversification of pigmentation pattern in clownfish and the developmental trajectories underlying white stripe formation. This sets up exciting perspectives for the study of color pattern diversity in coral reef fishes, for which an Evo/Devo approach was clearly underused until now. As suggested by our integrated study of clownfishes, the diversification of 
color patterns results from interplay among developmental, ecological, and behavioral processes.

\section{Methods}

Coding of striped patterns and associated polymorphisms Clownfishes were classified into four categories according to their striped pattern at the adult stage [52]: species without vertical stripe (group A) or species having one white vertical stripe on the head (group B) or two (head and trunk) (group C), or three (head, trunk, and caudal peduncle) (group D) (Additional file 1: Table S1). Four species (A. akallopisos, A. sandaracinos, A. perideraion, and $A$. pacificus) show an atypical pattern with a white line browsing the dorsal region [53]. This horizontal stripe was not taken into consideration in our study as it may come from a different developmental process. Three species (A. clarkii, A. melanopus, and A. polymnus) are polymorphic with two different patterns observed in natural populations (Additional file 1: Table S1). Accordingly, we repeated all comparative analyses using every combination of coding (i.e., eight combinations).

\section{Phylogeny, ancestral state reconstruction, and stochastic mapping}

We used stochastic character mapping [54] to infer possible histories of the stripe pattern and stripe pattern ontogenesis. The stochastic mapping and the ancestral state reconstruction was produced using the function make.simmap in the package phytools (version 0.5.38; [55]) for R [56]. We then sampled 10,000 character histories allowing the incorporation of the uncertainty associated with the timing of the transitions between morphological states. For the parameterization of make.simmap, we used the estimated ancestral state and the best model for the transition matrix from our empirical data. To assess the best model for the transition matrix, we fitted a model with equal rate of transition between states and a model with all rates different using the function ace in the R-package ape [57]. The likelihood of these two models was then compared using a likelihood ratio test, which suggested the use of unequal rates (see MuSSE results). Statistics for stripe morph histories were retrieved using the function describesimmap from the phytools R-package.

\section{Model of striped pattern evolution}

To test whether the evolution of striped patterns is not random in clownfishes, we compared transition rates between stripe morphs using the "multiple state speciation extinction" (MUSSE) method. MuSSE is an extension of the BiSSE maximum likelihood-based test, which is described in $[28,29]$. To test our hypotheses, we used the R-package diversitree [29] to compare the fit of four different models: (i) a model in which all transition rates vary independently, (ii) a model with an equal rate transition matrix (e.g., $q_{14}=q_{41}$ ), (iii) a model in which the loss or the acquisition of two stripes by evolutionary color shift is not allowed (i.e., $q_{31}=q_{41}=q_{42}=q_{13}=q_{14}=q_{24}=0$ ), and (iv) a model combining the constrains of model (ii) and model (iii). In order to reduce the number of parameters, every model assumed that the speciation rates $(\lambda)$ and the extinction rates $(\mu)$ are equal among stripe morphs. Moreover, we corrected for incompletely resolved phylogeny without specifying the state of missing species and we assumed that the missing species are randomly distributed on the phylogenetic tree. The fit of models was compared using sample-size-corrected Akaike's Information Criterion (AICc) scores and weights [58]. A $\triangle \mathrm{AICc}$ value of 4 or more was taken as an indication of support for one model over the others [58].

\section{Ecological and morphological data}

Our morphological analysis includes body elongation, body size, and dorsal fin morphologies. Maximum body size was retrieved from Fishbase [59]. Body elongation and dorsal fin morphology quantifications were determined using pictures of Amphiprion sp. individuals found in Fishbase [59] or nicely provided by J.E. Randall (http://pbs.bishopmuseum.org/images/JER/) and J. Williams. Others were studied in the marine vertebrate collections of S. Planes (CRIOBE) and Museum of Natural National History (MNHN). This analysis includes 22 species over the 30 described species (Additional file 1: Table S1). The species A. chagosensis, A. latezonatus, A. leucokranos, A. mccullochi, A. pacificus, and A. sebae are not studied here because of the lack of individuals for quantifications. To quantify body elongation, we used the ratio between body height and the standard length. In order to describe the ingression between the anterior and the posterior lobe of the dorsal fin, we calculated two indexes based on the length of the third dorsal spine (11), the length of the most posterior spine (12), the length of the longest soft ray (lr), and the length of the dorsal fin (L). Anterior and posterior lobe morphology indexes correspond to (11-l2)/L and (lr-12)/L, respectively (Fig. 6e). Ecological data (i.e., number of host sea anemones) were retrieved from FishBase [59] and the primary literature $[33,60]$.

\section{Relationships between striped patterns and fish morphology}

Body size and body elongation describe the overall fish shape, and it has been shown to be linked with swimming performance and with adaption towards different macro-habitats [35, 36]. Dorsal fins are associated with stability and thrust [61], and their shape may thus provide information on the swimming behavior of clownfishes. We predicted that the striped pattern of clownfishes is 
related to ecological and behavioral differences, and thus, phylogenetic generalized least-squares (PGLS) regressions using a Brownian motion model were used to test for correlated evolutionary relationships between the number of stripes and the forms of fish body and dorsal fin. PGLS analyses were performed in the R-package geiger (version 2.0.6; [62]).

\section{Species recognition hypothesis}

The composition of clownfish communities at eight locations was retrieved from Camp et al. [38], and we counted the number of identical striped pairs within each of the eight communities. To test if color similarity among sympatric species was less than would be expected by chance, we generated 9999 random communities by creating random communities using the function randomizeMatrix [63] from the R-package picante (version 1.6.2; [63]). Variation of species morph pool due to color polymorphism was also included in the generated communities. Then, we compared the number of identical species in each random community to the number in the natural community by a binomial test.

\section{Larval rearing and observation of color ontogenesis}

A. ocellaris and A. frenatus were maintained at $26^{\circ} \mathrm{C}$ in separate $60-\mathrm{L}$ aquaria. Breeding pairs laid egg clutches on the underside of a terracotta pot placed in their aquarium. On the night of hatching ( 9 days post laying, $26{ }^{\circ} \mathrm{C}$ ), egg clutches were transferred from the parental aquarium to a $30-\mathrm{L}$ larval rearing aquarium. Larvae were fed rotifers (Brachionus plicatilis) at 10 individuals per milliliter three times a day for the first 7 days. The ratio of Artemia nauplii to rotifers was increased each day until larvae were fed only five individuals of Artemia nauplii per milliliter from day 7 . From day 7 until day 20, larvae are euthanized or anesthetized in MS222 at $200 \mathrm{mg} / \mathrm{L}$ and $100 \mathrm{mg} / \mathrm{L}$, respectively, in filtered aquarium water and photographed under a stereomicroscope. At least three larvae per species per day of development are studied.

\section{Drug treatment of larvae}

TAE684 (NVP-TAE684) (HY-10192, MedChem Express), a specific inhibitor of Ltk and Alk [31], was diluted in dimethylsulphoxyde (DMSO; Sigma-Aldrich Louis, MI, USA) to a final concentration of $6 \mathrm{mM}$. Larvae were treated from 5 until $18 \mathrm{dph}$ in $0.005 \%$ DMSO with $0.3 \mu \mathrm{M}$ or $0.6 \mu \mathrm{M}$ TAE684 or without (controls). For each condition, five larvae were treated in $500-\mathrm{mL}$ fish medium in a beaker (in total 20 individuals per conditions). One hundred milliliters of solution was changed every day.

\section{Additional files}

Additional file 1: Table S1. Dataset list of studied species. Dataset list of studied species, with relative coding based on their color pattern [52], color pattern ontogenesis [64-72], and number of host sea anemones [33] and with quantifications of dorsal fin shape and body elongation. Photographs of specimens for quantifications of dorsal fin shape and body elongation were obtained from online databases (FishBase and "J. E. Randall's Fish Photos" http://pbs.bishopmuseum.org/images/JER/), or nicely provided by J. Williams. Others were studied in the marine vertebrate collections of S. Planes (CRIOBE) and the Museum of Natural National History (MNHN). Excel document 19 ko. (XLSX 18 kb)

Additional file 2: Figure S1. Successive caudo-rostral loss of stripes during evolution is independent of clownfish color polymorphism. Phylogenetic trees of clownfishes from Litsios et al. (2014) [26] with a summary map of white stripe number histories generated through stochastic character mapping. Here, all the combinations of species color pattern polymorphism have been taken into account. Interestingly, all these traits mapping show that the diversification of white striped pattern is a history of loss from an ancestral clownfish having three stripes and that these losses occurred in a progressive fashion in a caudal to rostral sequence. Circles at the tips of the tree indicate each species striped pattern and circles at every internal nodes give probabilities of ancestral striped pattern. PDF document 287 ko. (PDF $286 \mathrm{~kb}$ )

Additional file 3: Table S2. Summary statistics of the stochastic mapping. Summary statistics of the stochastic mapping (10,000 simulations), assuming that all transition rates (q) among stripe morphs are free to vary. Morphs are coded as followed: species without vertical stripe (A), species having one white vertical stripe on the head (B), species having two white vertical stripes (head and trunk) (C), species having three white vertical stripes (head, trunk, and caudal peduncle) (D). Results are provided for every combination of coding. Word document 13 ko. (DOCX $12 \mathrm{~kb}$ )

Additional file 4: Table S3. Model fitting of the four striped pattern evolutionary models. Model fitting of the four striped pattern evolutionary models, using the different coding of color patterns detailed in Additional file 1: Table S1. Models are ranked from best to worst, according to AIC scores and Akaike weights (wtAIC). $\triangle \mathrm{AIC}$ scores indicate the difference between the candidate model and the best-fitting model. According to [58], a $\Delta$ AICc value of 4 or more was taken as an indication of support for one model over the other following. Matrices of transition rates (q) among stripe morphs are described for the four evolutionary models. Speciation and extinction rates are assumed to be equal among morphs. See text for details about models and Additional file 1: Table S1 for color coding. Word document $14 \mathrm{ko}$. (DOCX $13 \mathrm{~kb}$ )

Additional file 5: Table S4. Relationship between the number of vertical white stripes and eco-morphological variables. Results from PGLS analyses. $X$ and $Y$ refer to the variables in the linear regression model. See Additional file 1 : Table 51 for color coding. Word document 14 ko. (DOCX 13 kb)

Additional file 6: Figure S2. Lobe morphology index of dorsal fin of clownfish species. Graphs representing the quantifications of posterior (a-b) and anterior (c-d) lobe morphology index in each species. In each graph, we presented the mean from the smallest to the biggest for each stripe morph. (a-c) Graphs represent the mean of posterior (a) and anterior (b) lobe morphology index (dots). Error bars indicate the standard deviation. (b-d) Boxplots of the mean of posterior (c) and anterior (d) lobe morphology index. Boxes extend from the 25 th to 75th percentiles, whereas whiskers go down to the smallest value and up to the largest. Median data are indicated by horizontal line within each box. PDF document 67 ko. (PDF 66 kb)

\section{Acknowledgements}

We thank V. Logeux, N. Trouillard, and P. Romans for fish husbandry and G. R. Allen, J. E. Randall, J. Williams, S. Planes, and the Museum of Natural History of Paris (MNHN) for sharing pictures or clownfish collections. We also thank N. Salamin, M. Besson, and T. Lorin for the critical discussions concerning the manuscript. 


\section{Funding}

Our clownfish husbandry has been supported by the CNRS (interdisciplinarity actions) as well as EMBRC-France, whose French state funds are managed by the ANR within the Investments of the Future program under reference ANR-10-INBS-02. BF was funded by the "Fonds National de la Recherche Scientifique of Belgium" (F.R.S-FNRS) and the Belgian Science Policy Office (Belspo).

\section{Availability of data and materials}

The datasets supporting the conclusions of this article are included within the article and its additional files.

\section{Authors' contributions}

PS, VL, and BF conceived the study and performed the research. PS performed all the experiments on clownfish development and TAE treatment. PS collected the data with the help of NR and DL. OS managed the rearing of clownfish. BF performed all comparative analyses. PS, VL, and BF led the writing of the manuscript with critical contributions from all authors. All authors read and approved the final manuscript.

\section{Ethics approval}

We have approval for these experiments from the C2EA - 36 Ethics Committee for Animal Experiment Languedoc-Roussillon (CEEA-LR), number A6601601. We have an approval number of premises for animal testing issued by the Regional Directorate of Food, Agriculture and Forestry of Occitania and the Departmental Directorate of Protection of Populations of the Pyrenees Orientales. This number is A6601601. The animals were raised in our structure from breeding stock. The experimental protocols were based on the regulations in force in France (Articles R214-87 to R214-137 of the Rural Code), updated by Decree 2013-118 and by five decrees dated February 1, 2013, and published on February 7, 2013, pursuant to Directive 2010/63/EU. This regulation is under the responsibility of the Ministry of Agriculture.

\section{Consent for publication}

Not applicable.

\section{Competing interests}

The authors declare that they have no competing interests.

\section{Publisher's Note}

Springer Nature remains neutral with regard to jurisdictional claims in published maps and institutional affiliations.

\section{Author details}

'Observatoire Océanologique de Banyuls-sur-Mer, UMR CNRS 7232 BIOM, Sorbonne Université Paris, 1, Avenue Pierre Fabre, 66650 Banyuls-sur-Mer, France. ${ }^{2}$ Aquarium de Canet-en-Roussillon, 2 Boulevard de la Jetée, 66140 Canet-en-Roussillon, France. ${ }^{3}$ EPHE-UPVD-CNRS, USR3278 CRIOBE, PSL Research University, BP 1013, 98729 Papetoai, Moorea, French Polynesia. ${ }^{4}$ Laboratory of Functional and Evolutionary Morphology, FOCUS, University of Liège, Liège, Belgium.

Received: 28 June 2018 Accepted: 2 August 2018

Published online: 05 September 2018

\section{References}

1. Brakefield PM. Evo-devo and accounting for Darwin's endless forms. Philos Trans R Soc Lond B Biol Sci. 2011;366:2069-75.

2. Hoekstra HE. Genetics, development and evolution of adaptive pigmentation in vertebrates. Heredity (Edinb). 2006;97:222-34.

3. Miyazawa S, Okamoto M, Kondo S. Blending of animal colour patterns by hybridization. Nat Commun. 2010;1:66.

4. Watanabe M, Kondo S. Changing clothes easily: Connexin41.8 regulates skin pattern variation. Pigment Cell Melanoma Res. 2012;25:326-30.

5. Teyssier J, Saenko SV, Van Der Marel D, Milinkovitch MC. Photonic crystals cause active colour change in chameleons. Nat Commun. 2015;6:66368.

6. Saenko SV, Teyssier J, van der Marel D, Milinkovitch MC. Precise colocalization of interacting structural and pigmentary elements generates extensive color pattern variation in Phelsuma lizards. BMC Biol. 2013;11:105.
7. Bellwood DR, Goatley CHR, Bellwood O. The evolution of fishes and corals on reefs: form, function and interdependence. Biol Rev Camb Philos Soc. 2017;92:878-901.

8. Katzir BYG. Visual aspects of species recognition in the damselfish Dascyllus aruanus L. ( Pisces, Pomacentridae ). Anim Behav. 1981;29:842-9.

9. Lecchini D, Peyrusse K, Lanyon RG, Lecellier G. Importance of visual cues of conspecifics and predators during the habitat selection of coral reef fish larvae. C R Biol. 2014;337:345-51.

10. Longley $\mathrm{WH}$. Observations upon tropical fishes and inferences from their adaptative coloration. Proc Natl Acad Sci U S A. 1916;2:733-7.

11. Cott HB. Adaptive coloration in animals. London: Methuen; 1940. p. 1-602.

12. Morris M, Rios-Cardenas O, Darrah A. Female mimicry and an enhanced sexually selected trait: what does it take to fool a male? Behaviour. 2010;147:1443-60.

13. Ehrlich PR. The population biology of coral reef fishes. Annu Rev Ecol Syst. 1975;6:211-47.

14. Gagliano M. On the spot: the absence of predators reveals eyespot plasticity in a marine fish. Behav Ecol. 2008:19:733-9.

15. Kelley JL, Fitzpatrick JL, Merilaita S. Spots and stripes: ecology and colour pattern evolution in butterflyfishes. Proc R Soc B. 2013;280:20122730.

16. Parichy DM. Evolution of Danio pigment pattern development. Heredity (Edinb). 2006:97:200-10.

17. Carroll SB. Evo-Devo and an expanding evolutionary synthesis: a genetic theory of morphological evolution. Cell. 2008;134:25-36.

18. Parichy DM, Spiewak JE. Origins of adult pigmentation: diversity in pigment stem cell lineages and implications for pattern evolution. Pigment Cell Melanoma Res. 2015;28:31-50.

19. Patterson LB, Bain EJ, Parichy DM. Pigment cell interactions and differential xanthophore recruitment underlying zebrafish stripe reiteration and Danio pattern evolution. Nat Commun. 2014;5:5299.

20. Quigley IK, Manuel JL, Roberts RA, Nuckels RJ, Herrington ER, EL MD, et al. Evolutionary diversification of pigment pattern in Danio fishes: differential fms dependence and stripe loss in D. albolineatus. Development. 2005;132:89-104.

21. Frédérich B, Mills SC, Denoël M, Parmentier E, Brié C, Santos R, et al. Colour differentiation in a coral reef fish throughout ontogeny: habitat background and flexibility. Aquat Biol. 2010;9:271-7.

22. Colleye O, Iwata E, Parmentier E. Clownfishes. In: Frédérich B, Parmentier E, editors. Biology of damselfishes. Boca Raton: CRC Press; 2016. p. 246-66.

23. Cooper WJ, Santini F. A revised damselfish taxonomy with a description of the tribe Microspathodontini (Giant damselfishes). In: Frédérich B, Parmentier $E_{i}$ editors. Biology of damselfishes. Boca Raton: CRC Press; 2016. p. 13-30.

24. Berumen ML, Walsh HJ, Raventos N, Planes S, Jones GP, Starczak V, et al. Otolith geochemistry does not reflect dispersal history of clownfish larvae. Coral Reefs. 2010;29:883-91.

25. Seehausen O, Mayhew PJ, Van AJJM. Evolution of colour patterns in East African cichlid fish. J Evol Biol. 1999;12:514-34.

26. Litsios G, Pearman PB, Lanterbecq D, Tolou N, Salamin N. The radiation of the clownfishes has two geographical replicates. J Biogeogr. 2014;41:2140-9.

27. Litsios G, Salamin N. Hybridisation and diversification in the adaptive radiation of clownfishes. BMC Evol Biol. 2014;14:245.

28. Maddison WP, Midford PE, Otto SP. Estimating a binary character's effect on speciation and extinction. Syst Biol. 2007;56:701-10.

29. Fitzjohn RG. Diversitree: comparative phylogenetic analyses of diversification in R. Methods Ecol Evol. 2012;3:1084-92.

30. Skold HN, Aspengren S, Cheney KL, Wallin M. Fish Chromatophores-from molecular motors to animal behavior. Int Rev Cell Mol Biol. 2016:321:171-219.

31. Fadeev A, Krauss J, Singh AP, Nüsslein-Volhard C. Zebrafish leucocyte tyrosine kinase controls iridophore establishment, proliferation and survival. Pigment Cell Melanoma Res. 2016;29:284-96.

32. Elliott JK, Mariscal RN. Coexistence of nine anemonefish species: differential host and habitat utilization, size and recruitment. Mar Biol. 2001;138:23-36.

33. Litsios G, Sims CA, Wüest RO, Pearman PB, Zimmermann NE, Salamin N. Mutualism with sea anemones triggered the adaptive radiation of clownfishes. BMC Evol Biol. 2012;12:212.

34. Kondo $\mathrm{S}$, Shirota $\mathrm{H}$. Theoretical analysis of mechanisms that generate the pigmentation pattern of animals. Semin Cell Dev Biol. 2009;20:82-9.

35. Langerhans RB, Layman CA, Langerhans AK, Dewitt TJ. Habitat-associated morphological divergence in two Neotropical fish species. Biol J Linn Soc. 2003:80:689-98.

36. Aguilar-Medrano R, Frédérich B, Barber PH. Modular diversification of the locomotor system in damselfishes (Pomacentridae). J Morphol. 2016;277: 603-14. 
37. Hone DWE, Naish D. The "species recognition hypothesis" does not explain the presence and evolution of exaggerated structures in non-avialan dinosaurs. J Zool. 2013;290:172-80.

38. Camp EF, Hobbs J-PA, De Brauwer M, Dumbrell AJ, Smith DJ. Cohabitation promotes high diversity of clownfishes in the coral triangle. Proc R Soc B. 2016;283:20160277.

39. Frohnhöfer HG, Krauss J, Maischein H-M, Nüsslein-Volhard C. Iridophores and their interactions with other chromatophores are required for stripe formation in zebrafish. Development. 2013;140:2997-3007.

40. Watanabe M, Kondo S. Is pigment patterning in fish skin determined by the Turing mechanism? Trends Genet. 2015;31:88-96.

41. Kondo S, Miura T. Reaction-diffusion model as a framework for understanding biological pattern formation. Science. 2010;329:1616-20.

42. Inaba M, Yamanaka H, Kondo S. Pigment pattern formation by contactdependent depolarization. Science. 2012;335(6069):677.

43. van Eeden FJ, Granato M, Schach U, Brand M, Furutani-Seiki M, Haffter P, et al. Genetic analysis of fin formation in the zebrafish, Danio rerio. Development. 1996;123:255-62.

44. Kondo S, Asai R. A reaction diffusion wave on the skin of the marine angelfish Pomacanthus. Nature. 1995;376:765-8.

45. Parichy DM. Temporal and cellular requirements for Fms signaling during zebrafish adult pigment pattern development. Development. 2003;130:817-33.

46. Mo ES, Cheng Q, Reshetnyak AV, Schlessinger J, Nicoli S. Alk and Ltk ligands are essential for iridophore development in zebrafish mediated by the receptor tyrosine kinase Ltk. Proc Natl Acad Sci U S A. 2017;114:12027-32.

47. Cuthill IC, Stevens M, Sheppard J, Haddocks T, Párraga CA, Troscianko TS Disruptive coloration and background pattern matching. Nature. 2005;434: $72-4$.

48. Lyon BE, Montgomerie R. Sexual selection is a form of social selection. Philos Trans R Soc Lond B Biol Sci. 2012:367:2266-73.

49. Buston P. Social hierarchies: size and growth modification in clownfish. Nature. 2003:424:145-6.

50. Colleye O, Frederich B, Vandewalle P, Casadevall M, Parmentier E. Agonistic sounds in the skunk clownfish Amphiprion akallopisos: size-related variation in acoustic features. J Fish Biol. 2009;75:908-16.

51. Colleye O, Parmentier E. Overview on the diversity of sounds produced by clownfishes (Pomacentridae): importance of acoustic signals in their peculiar way of life. PLoS One. 2012;7(11):e49179.

52. Fautin DG, Allen GR. Anemone fishes and their host sea anemones. Perth: Ed: Western Australian Museum; 1994

53. Allen GR. The anemonefishes: their classification and biology. Neptune City: T.F.H. Publications; 1972

54. Huelsenbeck JP, Nielsen R, Bollback JP. Stochastic mapping of morphological characters. Syst Biol. 2003;52:131-58.

55. Revell LJ. Phytools: an R package for phylogenetic comparative biology (and other things). Methods Ecol Evol. 2012;3:217-23.

56. R: a language and environment for statistical computing [Internet]. Vienna Austria: R Foundation for Statistical Computing; 2008. Available from: http://www.r-project.org.

57. Paradis E, Claude J, Strimmer K. APE: analyses of phylogenetics and evolution in R language. Bioinformatics. 2004:20:289-90.

58. Burnham KP, Anderson DR. Model selection and multimodel inference: a practical information-theoretic approach. New York: Springer; 2002.

59. Froese R, Pauly D. Fishbase. 2018. www.fishbase.org. Accessed Jan 2018.

60. Fautin DG, Allen GR. Field guide to anemonfishes and their host sea anemones. Perth: West. Aust. Museum; 1997. p. 160.

61. Drucker EG, Lauder GV. Locomotor function of the dorsal fin in teleost fishes: experimental analysis of wake forces in sunfish. J Exp Biol. 2001;204: 2943-58.

62. Pennell MW, Eastman JM, Slater GJ, Brown JW, Uyeda JC, Fitzjohn RG, et al. Geiger v2.0: an expanded suite of methods for fitting macroevolutionary models to phylogenetic trees. Bioinformatics. 2014;30:2216-8.

63. Kembel SW, Cowan PD, Helmus MR, Cornwell WK, Morlon H, Ackerly DD, et al. Picante: $R$ tools for integrating phylogenies and ecology. Bioinformatics. 2010;26:1463-4

64. Dhaneesh KV, Nanthini Devi K, Ajith Kumar TT, Balasubramanian T, Tissera K Breeding, embryonic development and salinity tolerance of skunk clownfish Amphiprion akallopisos. J King Saud Univ Sci. 2012;24:201-9.

65. Richardson. Descriptions of the colour patterns of juvenile anemonefishes (Pomacentridae: Amphiprion) from New South Wales and the Lord HoweNorfolk Island Region. Proc. Linn. Soc. N.S.W. 1998;120(81):86.
66. Van Der Elst R, Borchert P. A guide to the common sea fishes of Southern Africa. 3rd ed. Cape Town: Struik Publishers; 1993.

67. Kumar TTA, Gopi M, Dhaneesh KV, Vinoth R, Ghosh S, Balasubramanian T, et al. Hatchery production of the clownfish Amphiprion nigripes at Agatti island, Lakshadweep, India. J Environ Biol. 2012;33:623-8.

68. Dhaneesh KV, Ajith Kumar TT, Swagat G, Balasubramanian T. Breeding and mass scale rearing of clownfish Amphiprion percula: feeding and rearing in brackishwater. Chin J Oceanol Limnol. 2012;30:528-34.

69. Ignatius B, Rathore G, Jagadis I, Kandasami D, Victor ACC. Spawning and larval rearing technique for tropical clownfish Amphiprion sebae under captive condition. J Agric Trop. 2001;16:241-9.

70. Madhu K, Madhu R, Gopakumar G, Sasidharan CS, Venugopalan KM Breeding, larval rearing and seed production of maroon clown Premnas biaculeatus under captive conditions. Mar Fish Inf Serv Tech Ext Ser. 2006; 190:124-9.

71. Michael SW. Damselfishes and Anemonefishes: the complete illustrated guide to their identification, behaviors, and captive care. Neptune City: T.F. H. Publications; 2008.

72. Salis P, Roux N, Lecchini D \& Laudet V. The post-embryonic development of Amphiprion perideraion reveals a decoupling between morphological and pigmentation changes. Cybium (accepted).

Ready to submit your research? Choose BMC and benefit from:

- fast, convenient online submission

- thorough peer review by experienced researchers in your field

- rapid publication on acceptance

- support for research data, including large and complex data types

- gold Open Access which fosters wider collaboration and increased citations

- maximum visibility for your research: over $100 \mathrm{M}$ website views per year

At $\mathrm{BMC}$, research is always in progress.

Learn more biomedcentral.com/submissions 